Internist 2014 · 55:5-6

DOI 10.1007/s00108-013-3309-5

Online publiziert: 15. Januar 2014

(c) Springer-Verlag Berlin Heidelberg 2014

\author{
J. Mössner ${ }^{1} \cdot$ W. Hiddemann ${ }^{2}$ \\ ${ }^{1}$ Department für Innere Medizin, Neurologie und Dermatologie, Klinik und Poliklinik \\ für Gastroenterologie und Rheumatologie, Universitätsklinikum Leipzig \\ ${ }^{2}$ Medizinische Klinik und Poliklinik III, Klinikum der Universität München, Campus Großhadern
}

\title{
Gastrointestinale Tumoren
}

Gastrointestinale Tumoren gehören zu den häufigsten Malignomen. So steigt die Inzidenz des Barrett-Karzinoms des Ösophagus in den westlichen Industrienationen deutlich an. Obgleich die Pathogenese und Epidemiologie dieses Tumors noch Fragen offen lässt, ist anzunehmen, dass die Inzidenzzunahme mit der $\mathrm{Zu}$ nahme der gastroösophagealen Refluxkrankheit korreliert. Überraschend ist in diesem Zusammenhang, dass viele Patienten, die erst die Dysphagie zum Arzt führt, nicht über vorbestehende Refluxbeschwerden klagen. Schützt das BarrettEpithel vor der Symptomatik, da es Säure eher toleriert als das Plattenepithel? Für auf die Mukosa begrenzte Tumoren ist die endoskopische Mukosaresektion das Therapieverfahren der Wahl. Leider werden sowohl Platten- als auch Adenokarzinome oft erst in einem fortgeschrittenen Stadium bei dann auftretender Dysphagie diagnostiziert. Hier muss im Tumorboard besprochen werden, ob die Therapie der ersten Wahl in einer neoadjuvanten Radiochemotherapie mit nachfolgender Resektion oder "nur" in einer definitiven Radiotherapie besteht. In der palliativen Situation stellt sich die Frage, ob bei Multimorbidität eine Chemotherapie überhaupt noch indiziert ist. Bei Dysphagie ermöglicht die endoskopische Platzierung eines Stents eine Verbesserung der Lebensqualität. Eine Bestrahlung ,,von innen" (Afterloading) ist eher in den Hintergrund getreten. M. Stahl aus Essen und T. Ruhstaller aus St. Gallen setzen in ihrem Beitrag den Fokus auf die Möglichkeiten der Kombinationstherapien, z. B. der trimodalen Therapie mit Radiochemotherapie gefolgt von einem operativen Eingriff.

Die Inzidenz des Magenkarzinoms nimmt ab. Hier liegt die Korrelation mit dem Rückgang der Helicobacter-pylori-
Infektion auf der Hand. Dennoch ist das Magenkarzinom kein seltener Tumor. Die unzureichende Kostenerstattung einer ambulant durchgeführten Ösophagogastroduodenoskopie mag mit ein Grund dafür sein, dass wir in Deutschland im Gegensatz zu Japan das Magenfrühkarzinom viel zu selten diagnostizieren.

\section{》) Magenkarzinome werden in Deutschland viel zu selten in einem frühen Stadium diagnostiziert}

Wir stellen die Indikation zur Ösophagogastroduodenoskopie wohl zu selten, endoskopieren vielleicht zu hastig und übersehen dabei Frühkarzinome. In der Behandlung von Tumoren, die mit kurativer Intention operabel sind, hat sich mittlerweile die perioperative Chemotherapie etabliert. In der palliativen Situation bieten sich die Optionen unterschiedlicher Chemotherapieregime. Von Interesse ist außerdem der HER2-Rezeptor-Status, da der zusätzliche Einsatz des monoklonalen Antikörpers Trastuzumab eine weitere Therapieoption darstellt. F. Lordick u. A. Hoffmeister aus Leipzig stellen das gesamte Spektrum der Diagnostik und Therapie beim Karzinom des gastroösophagealen Übergangs und dem Magenkarzinom sehr pointiert dar.

Eine chronische Leberentzündung ob durch Alkohol, eine chronische Hepatitis-B- bzw. -C-Virus-Infektion oder eine Fettleberhepatitis verursacht - ist der wichtigste Risikofaktor für die Entstehung eines hepatozellulären Karzinoms (HCC). Angesichts der zunehmenden Übergewichtsproblematik und des fortgesetzt hohen Alkoholmissbrauchs in unserer Gesellschaft werden wir das HCC künftig wohl noch häufiger diagnostizieren. Es ist daher nicht nur ein Problem in Ländern mit einer hohen Prävalenz chronischer Hepatitis-B- oder -C-Virusinfektionen. Wie M.A. Wörns, R. Klöckner, A. Weinmann u. P.R. Galle aus Mainz in ihrem Beitrag zeigen, ist das Spektrum der stadienabhängigen Therapiemöglichkeiten groß: Es reicht vom Einsatz des Tyrosinkinaseinhibitors Sorafenib über die CT- oder sonographiegesteuerte Radiofrequenzablation und transarterielle Chemoembolisation (TACE) bis zur selektiven intraarteriellen Radiotherapie (SIRT). $\mathrm{Ob}$ bei fortgeschrittenem HCC das Tumor-Debulking mittels TACE oder SIRT wirklich die Lebensqualität verbessert oder das Leben verlängert, muss in Studien geklärt werden. In der Regel entsteht das HCC auf dem Boden einer Leberzirrhose. Die Transplantation ist daher tatsächlich der einzige kurative Therapieansatz. Da die HCC-Rezidivrate nach Transplantation mit dem Stadium des Tumors vor Transplantation korreliert, wird bei der Organallokation die strikte Einhaltung der Mailand-Kriterien gefordert. Die komplexen stadienabhängigen Therapieoptionen bis hin zur Lebertransplantation legen nahe, diese Patienten nur in erfahrenen Zentren zu behandeln.

\section{\) Das Pankreaskarzinom bleibt eines der traurigsten Kapitel der Inneren Medizin}

Die molekulare Pathogenese des Pankreaskarzinoms wird zunehmend verstanden. Bezüglich der Früherkennung und Heilung ist dennoch immer noch kein Licht am Ende des Tunnels zu erkennen. Der Tumor bleibt eines der traurigsten Kapitel der Inneren Medizin. Es gibt kei- 
ne kosteneffizienten Verfahren mit hoher Sensitivität und Spezifität zur Frühdiagnostik und zum Screening. Allenfalls bei den selteneren Risikogruppen, z. B. mit familiärer Belastung, wird der Einsatz der Endosonographie diskutiert. Nach „kurativer" Resektion ist Gemcitabin als adjuvante Therapie etabliert. In der palliativen Chemotherapie bieten sich jetzt mehr Möglichkeiten als Gemcitabin allein:

- Kombination der Chemotherapeutika Oxaliplatin und Irinotecan mit Folinsäure und 5-Fluorouracil (FOLFIRINOX)

- Einsatz des Tyrosinkinaseinhibitors Erlotinib zusätzlich zu Gemcitabin

- In Nanopartikeln verpacktes Paclitaxel, eine neue galenische Form dieses Taxans

Leider bleibt die gewonnene Lebensverlängerung, wenn überhaupt, auf wenige Monate beschränkt. U. Pelzer, M. Sinn, J. Stieler u. H. Riess aus Berlin stellen die komplexen Therapiemöglichkeiten dar und betonen, dass bei Patienten in noch gutem Allgemeinzustand FOLFIRINOX trotz seiner höheren Nebenwirkungsrate in der palliativen Therapie wahrscheinlich die Kombination der ersten Wahl sein wird.

Einzig das kolorektale Karzinom, bei der Frau der zweithäufigste, beim Mann der dritthäufigste Tumor, bietet Anlass zur Hoffnung. Soweit die Akzeptanz der Vorsorgekoloskopie steigt, wird eine deutliche Senkung der Sterblichkeit zu erreichen sein, da sich ungefähr 80\% der Karzinome erst langsam aus gutartigen Adenomen entwickeln. DNA-Stuhltests zur Detektion verschiedener mutierter Onkogene und Tumorsuppressorgene in vom Karzinom abgeschilferten Epithelien sind noch nicht praxisreif. Die Therapiemöglichkeiten in fortgeschrittenen Stadien sind heute komplex. So wird im interdisziplinären Tumorboard beispielsweise besprochen, ob durch neoadjuvante Chemotherapie Leber- und/oder Lungenmetastasen noch resektabel werden. So werden alle genannten Tumoren molekulargenetisch immer differenzierter charakterisiert. Allein beim kolorektalen Karzinom gibt es mehrere Subtypen. Die Kenntnis der Molekulargenetik und Epigenetik hat bereits Einfluss auf die Therapie. Hier darf die Wirkungslosigkeit einer Therapie mit monoklonalen Antikörpern gegen den „epidermal growth factor receptor" genannt werden, wenn verschiedene K-RAS-Mutationen vorliegen. D.P. Modest u. V. Heinemann aus München stellen die Therapiemöglichkeiten bei bereits metastasierten kolorektalen Karzinomen vor.

Es besteht Grund zur Hoffnung, dass bei besserer Kenntnis der molekulargenetischen Veränderungen des Tumors die zielgerichtete Therapie mit Biologika weiter verbessert wird. So bleibt auch zu hoffen, dass neben monoklonalen Antikörpern gegen Wachstumsfaktorrezeptoren und Tyrosinkinaseinhibitoren („small molecules“, Prototyp Imatinib) weitere Substanzen gefunden werden, die gezielt in die Signaltransduktionskaskaden eingreifen. Denkbar wäre eine Hemmung von "small interfering RNA“ (siRNA). Die Erforschung der molekularen Pathogenese der genannten Neoplasien sowie die Suche nach Präventionsstrategien und nach Methoden der Frühdiagnose werden uns noch viele Jahre begleiten. Das zunehmend komplexe Spektrum der Therapie legte nahe, allein die- sem Thema einen Schwerpunkt in Der Internist zu widmen.

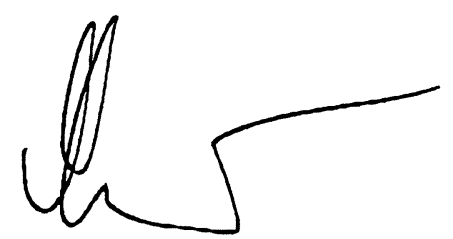

J. Mössner

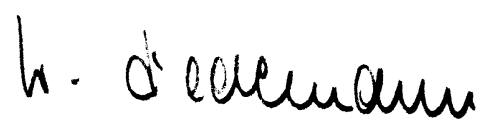

W. Hiddemann

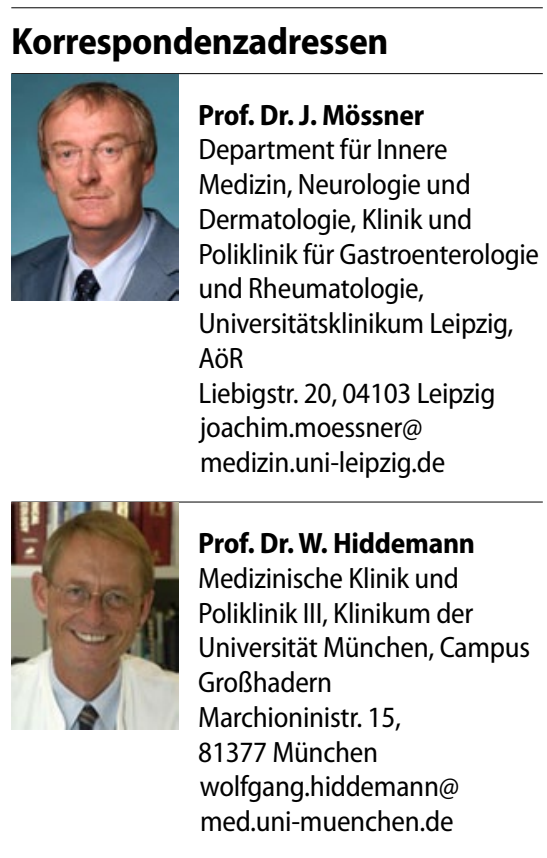

Einhaltung ethischer Richtlinien

Interessenkonflikt. J. Mössner und W. Hiddemann geben an, dass kein Interessenkonflikt besteht. 